\title{
The nse predictive value in pr development
}

\author{
D.I. Tashkhanova ${ }^{1}$, M.Kh. Karimova ${ }^{2}$ \\ 1,2 "Republican specialized scientific practical-medical center for eye microsurgery" state university
}

\begin{abstract}
Cardiovascular, respiratory, and nervous systems neonatal pathology in premature babies leads to systemic and organ hypoxia, which is one of the main risk factors for premature retinopathy (PR) development. Among the certain neuron-specific proteins, the most studied and adequately characterizing the blood-brain barrier (BBB) proper membrane function is neuro-specific enolase (NSE), which is currently used to diagnose acute conditions characterized by cerebral
\end{abstract} ischemia and brain hypoxia, occurring with impaired BBB function.

Research purpose is to evaluate the neuro-specific enolase activity in patients with premature retinopathy. To conduct NSE concentration comparative analysis in the blood serum in 109 main and control groups' newborns, was carried out the competitive enzyme-linked immunosorbent assay (ELISA) method.

Research results. A comparative study of blood serum parameters based on NSE content revealed significant differences between the newborns groups depending on the gestational age and central nervous system damage degree.

Conclusion. NSE higher levels in children with premature retinopathy are not only BBB damage index, but also reflect the combined, CNS damage deeper nature (hypoxia, intoxication, neurodegeneration). This indicates that premature retinopathy develops against the circulatory retinal hypoxia background.

Key words: blood-brain barrier, hypoxia, neurospecific enolase, premature retinopathy

\section{Introduction}

Improvements in neonatal care have led to a progressive decrease in preterm infants mortality rate with extremely low and very low birth weight $(5,6)$. At the same time, the high frequency of damage to immature internal organs and premature infants' body systems in the postnatal period contributes to the chronic pathology formation in them and neuro cognitive development impairment in the future (4).

A premature baby is at a reactive oxygen species-induced diseases increased risk such as premature retinopathy (PR), which leads to visual disability. Blood flow violation in the premature baby eye membranes leads to tissue ischemia, its edema and hypoxia, with the retina being particularly affected $(2,8)$. One of the risk factors for PR development in newborns is circulatory retinal hypoxia, which develops as the cardiovascular, respiratory, and nervous systems neonatal pathology result in premature infants and leads to systemic and organ hypoxia (2).

In hypoxia, the brain damage incidence in newborns is $20-40 \%$, and in premature newborns it reaches $60-70 \%$ (3).

Perinatal hypoxia triggers processes leading to an increase in cell membranes permeability, the neurons and glial cells death due to necrosis and apoptosis. The oxidative damage accumulation causes the blood-brain (BB) and bloodophthalmic barriers (BOB) permeability violation. The increased BBB and BOB permeability emergence with a variety of damage to the nervous tissue (hypoxic-ischemic, traumatic, infectious genesis) leads to the neurospecific proteins entry into the blood $(1,2,3,7)$.

As BBB permeability index for diagnostic purposes, used myelin basic protein (MBP), neurospecific enolase (NSE) is the only currently known common index of all differentiated neurons, qualitative and quantitative determinations which in the cerebrospinal fluid or blood serum provide valuable information about the neuronal damage severity and BBB overall integrity violations, which characterizes the post ischemic brain damage degree (1).

Thus, the premature retinopathy etiology study, the most significant perinatal factors influence and their prognostic value on the disease and its outcomes course, the primary damage importance to the organ and system that induced its development, the search for early prognosis ways and this disease prevention, determined the study purpose.

\section{Research materials and methods}

To solve the set goals and objectives was carried out a comparative examination of 109 newborn children. The newborns gestational age varied from 26 to 40 weeks, the birth weight, depending on the gestational age, was within from 820 to 3550 grams, the body length was from 32 to $54 \mathrm{~cm}$. The boys were 43, 66 girls.

In the study course, all premature babies were divided into 3 groups depending on gestational age and the premature babies' retinopathy incidence:

Group A - 32 premature infants without retinopathy premature;

Group B - 22 premature infants without retinopathy premature;

The control group consisted of 55 newborns born at 38-40 weeks gestation.

To carry out NSE concentration comparative analysis in the blood serum of all newborns of the main and control groups of Uzbek nationality, a competitive ELISA method was carried out with standard kits from "Fugirebio" on ELISA semiautomatic "Platoscinn" from "Hospitex (Italy)"; 


\section{Research results}

A comparative study of blood serum parameters based on NSE content revealed significant differences between the newborns groups depending on the gestational age and the central nervous system damage degree.

Neuro-specific enolase content in the respondent newborns. (ng/ml)

\begin{tabular}{|c|c|c|c|}
\hline \multirow{2}{*}{ Groups } & \multicolumn{3}{|c|}{ Indicator in observation dynamic } \\
\cline { 2 - 4 } & $1^{\text {st }}$ day & $\mathbf{3 - 5}^{\text {th }}$ days & $\mathbf{2 8}^{\text {th }}$ days \\
\hline Main A group & $24,5 \pm 2,2^{*}$ & $63,6 \pm 8,3^{*}$ & $30,6 \pm 6,2^{*}$ \\
\hline Main B group & $22,7 \pm 2,6^{*}$ & $86,5 \pm 7,2^{*}$ & $52,2 \pm 7,7^{*}$ \\
\hline Control group & $6,7 \pm 0,8$ & $8,5 \pm 1,8$ & $5,4 \pm 2,7$ \\
\hline
\end{tabular}

Note: $*$ is significant difference $(\mathrm{p}<0.05)$ in $\mathrm{t}$ - criterion in relation to the control group

The NSE indices in the main group A and B in the first day of life were $24.5 \pm 2.2 \mathrm{~m} \mu \mathrm{g} / \mathrm{ml}$ and $22.7 \pm 2.6 \mathrm{~m} \mu \mathrm{g} / \mathrm{ml}$, respectively, which is significantly higher than newborns indicator in the control group, which is $6.7 \pm 0.8 \mathrm{~m} \mu \mathrm{g} / \mathrm{ml}$. However, its highest level was noted in the main A group, which is most likely associated with the central nervous system ischemic (CNS) lesions predominance in this group of children.

NSE increased level was noted on the 4th day of life in all groups children $94 \%$ cases when compared with the norm and averaged $63.6 \pm 8.3 \mathrm{m \mu g} / \mathrm{ml}$ was in group A premature infants, in premature babies in group B was $86.5 \pm 7.2 \mathrm{ng} / \mathrm{ml}$. The average level of this indicator on the 4 th day of life in healthy mature infants was $8.5 \pm 1.8 \mathrm{~m} \mu \mathrm{g} / \mathrm{ml}$. The study revealed a statistically significant increase in NSE level in all groups' children, especially in children with premature retinopathy.

It should be noted that, this protein indicator especially increases on the 4th day of life and rises from 2 to 4 times in comparison with the indicator at birth on the following days.

The revealed changes indicate deeper damage to BBB in children with premature retinopathy, which is due to the postponed hypoxia consequences.

A decrease in NSE on day 28 indicates a decrease in destructive processes in neurons. Observation in dynamics (26-28 days of life) showed that in both groups' children there was a decrease in serum NSE indices. The NSE level in premature infants with premature retinopathy by the end of the first month of life was $52.2 \pm 7.7 \mathrm{~m} \mu \mathrm{g} / \mathrm{ml}$ (in the second group was $30.6 \pm 6.2 \mathrm{m \mu g} / \mathrm{ml})$. According to the literature, NSE content in term infants' blood serum by the end of the first month of life is $2.2 \pm 0.4 \mathrm{m \mu g} / \mathrm{ml}$. With NSE initial determination in studied groups' children, the studied indicator dependence on gestational age was noted: the younger the gestational age, the higher the NSE scores which may indicate the BBB permeability dependence on structures maturity degree. It should be noted that in children with premature retinopathy, the NSE level remained prohibitively high during dynamic observation and did not reach the normative level by the first month end of life. A slower decrease in NSE in main group children is due to combined damage to BBB, and does not exclude the apoptosis processes presence, which is consistent with research data. Based on our data, we can conclude that with the NSE initial determination in studied groups' children, this indicator dependence on gestational age was noted: the younger the gestational age, the higher the NSE scores which may indicate the BBB permeability dependence on the structures maturity degree. Higher NSE levels in infants with premature retinopathy are not only BBB damage index, but also reflect the combined, the damage deeper nature to the central nervous system (CNS) (hypoxia, intoxication, neurodegeneration). This indicates that premature retinopathy develops against the circulatory retinal hypoxia background.

\section{Conclusion}

In newborns with PR, there is a significant increase in NSE in the blood, which depends on the child's gestational age, and can serve as hypoxic damage impairment and prognosis varying degrees informative index to the brain and retina in children with PR.

\section{REFERENCES}

1. Jukova I.A., Alifirova V.M., Jukova N.G. Neuron-specific enolase as a specific index of the neurodegenerative process // Siberian Medicine Bulletin. - 2011. - № 2. - p. 15-21.

2. Nikolaeva G.V., Guseva M.R., Beslaneeva M.B. The prevention and antioxidant therapy effectiveness Analysis in premature infants // Ophthalmology blletin.- 2012.- № 6.- p. 57-61. 
3. Salikhova K.Sh., Ishniyazova N.D., Abdurakhmanova F.T., Ibragimova S. T. Immuno-metabolic aspects of early adaptation and prediction of post-hypoxic conditions in newborns // Pediatrics. - 2012. - № 3-4. - p. 132-137.

4. Sakharova E.S., Keshishyan E.S., Alyamovskaya G.A. Prematurity as a medico-social health problem. Part 1.// Perinatology and Pediatrics Russian Bulletin.-2017;62(3).-p. 15-19.

5. Tereshchenko A.V. Ophthalmic care organization for children with premature retinopathy. Russian pediatric ophthalmology. 2015. № 3. - p. 7-11.

6. Shilova N.A., Kharlamova N.V., Fisyuk Yu.A. etc. The incidence and outcomes of retinopathy in deeply term infants in the context of specialized medical care// Perinatology and Pediatrics Russian Bulletin.-2018;63(5).-p. 51-54.

7. DaiH.Q. Changes of electro encephalographic background patterns and serum neuron specific enolase level sinneonates with hypoxic-ischemic encephalopathy / H.Q. Dai , Y.H. Luo // Zhongguo Dang Dai ErKeZaZhi. 2009. - Mar;11(3). - P. 173-6.

8. Lee J.W., Davis J.M. Future applications of antioxidants in premature infants // Curr. Opin. Pediatr.- 2011; 23: $2: 161-166$. 